ENCYCLOPEEDE Encyclopédie berbère

BERBERE

27 | 2005

27 | Kairouan - Kifan Bel-Ghomari

\title{
Kef el-Kérem
}

C. Roubet

\section{OpenEdition}

Journals

Édition électronique

URL : http://journals.openedition.org/encyclopedieberbere/1328

DOI : 10.4000/encyclopedieberbere.1328

ISSN : 2262-7197

\section{Éditeur}

Peeters Publishers

\section{Édition imprimée}

Date de publication : 1 août 2005

Pagination : 4128-4131

ISBN : 2-7449-0538-0

ISSN : 1015-7344

\section{Référence électronique}

C. Roubet, «Kef el-Kérem », Encyclopédie berbère [En ligne], 27 | 2005, document K35, mis en ligne le 01 juin 2011, consulté le 25 septembre 2020. URL : http://journals.openedition.org/encyclopedieberbere/ 1328 ; DOI : https://doi.org/10.4000/encyclopedieberbere.1328

Ce document a été généré automatiquement le 25 septembre 2020

(c) Tous droits réservés 


\section{Kef el-Kérem}

\section{Roubet}

1 À $35 \mathrm{~km}$ au sud-est de Tiaret, près de l'axe routier Tiaret-Aflou, au delà de l'agglomération de Nador (Trézel), se dressent les derniers chaînons telliens de l'Oranie orientale. Orientés SE/NW, les Djebels ben Nsoui (1 $474 \mathrm{~m}$ ) et Nador/Belvédère (1453 m) surplombent une zone de daïa et de grands chotts, formant les Hauts-Plateaux oranais. $\mathrm{Au}$ nord-ouest du Djebel Nador s'élève le relief tabulaire du Kef el-Kérem et jaillit la résurgence de l'Oued Sousselem (Cadenat, 1966, Carte $n^{\circ} 247$ de Trézel au 1/50 000è, 1958 ; Feuille $n^{\circ} 33$ P. de Tiaret au $1 / 200000^{\grave{e}}$, site $n^{\circ} 189$, p. 94-95).

2 Sur la rive droite de cet oued la station épipaléolithique du Kef el-Kérem occupe la pente nord-ouest du Kef. Les fouilles, pratiquées dès 1938 par P. Cadenat et G. Vuillemot (1944), livrèrent des informations culturelles originales, isolant le nouvel ensemble culturel de l'Ibéromaurusien et du Capsien d'Oranie, comme F. Doumergue l'avait pressenti (1898). Bien peu d'études suivirent. Mais, comme le besoin d'esquisser les principales tendances de l'Épi-paléolithique du Maghreb se faisait sentir, cet exemple, au particularisme régional affirmé, incita L. Balout (1955, p. 365-366) à nommer ce contexte : Kérémien. Dans la région de Tiaret d'autres sites vinrent plus tard conforter l'individualisation du Kérémien, sans toutefois l'imposer. Les principaux gisements sont le Bois de Pins (Bayle des Hermens 1959-1965), la Jumenterie (Bayle des Hermens et Tixier 1967-1972) et Torrich-Vigne Serrero (Bonneau et Vassot 1955 ; Cadenat 1966).

\section{Kérémien : unité culturelle épipaléolithique non ibéromaurusienne, non capsienne. Le site éponyme}

\section{Les fouilles}

3 Au Kef el-Kérem, P. Cadenat et G. Vuillemot retirent d'une longue tranchée d'un mètre d'épaisseur et de largeur, des dépôts in situ, uniformément cendreux, au contenu culturel homogène. Ils observent vers la base des accumulations ou brèches compactes, contrastant avec l'aspect pulvérulent et pierreux des couches supérieures et décident de les séparer. Ils notent que cette occupation de plein air, qui n'est pas une escargotière, 
est tronquée, qu'elle est privée de son dernier épisode, en raison de sa localisation et d'une forte érosion qu'aucun boisement ne freine, qu'elle s'étend au delà du secteur fouillé, protégée par les gradins rocheux du Kef, non loin d'un petit abri, resté vide.

\section{Le contenu archéologique}

4 Pour la première fois au Maghreb, dans un équipement lithique épipaléolithique, la dominance ne concerne pas l'armature mais le plus commun des outils : le grattoir.

5 La spécificité du Kérémien s'exprime avec netteté et nuance. Elle résulte de la conception même de cette industrie lithique, dépendante d'une finalité fonctionnelle originale, maintenue stable et dominante. Elle impose une structure lithique riche en éclats, éclats-laminaires (fig. 1), faible en lamelles : mixte en somme, ayant un groupe d'outils majeurs dont la fonction reste à définir, ce qui marginalise un peu les autres constituants. Certes, les armatures présentent des caractères originaux et une certaine diversité, cependant l'incidence comportementale - sur la chasse s.l. - qu'impliqua leur moins forte représentation parut d'emblée déroutante, mais très originale. P. Cadenat et G. Vuillemot (1944:56) insistent sur la place des grattoirs dans cet assemblage «ils submergent les autres types et se présentent sous des formes et des dimensions variées ». Viennent ensuite des microlithes, c'est-à-dire des lamelles à bord abattu, à cran, gibbeuse, à troncature, à pédoncule ou en pointe, de type Ounan, puis les armatures géométriques, telles le triangle scalène, l'isocèle et un nouveau trapèze à trois côtés retouchés, jusque-là inconnu. L'inventaire qui souligne la présence de racloirs (habituellement peu nombreux en contexte épipaléolithique), de coches et denticulés fréquents, insiste sur la rareté des burins, perçoirs et microburins (1944: 55-56 et 61 ; Cadenat, 1969-1972 : 129-132). 
Fig. 1. La Jumenterie (région de Tiaret, Algérie). Kérémien : 1, 2, 5 : racloirs ; $3,4:$ burins ; 6 à 8 : denticulés (d'après R. de Bayle des Hermens 1972).

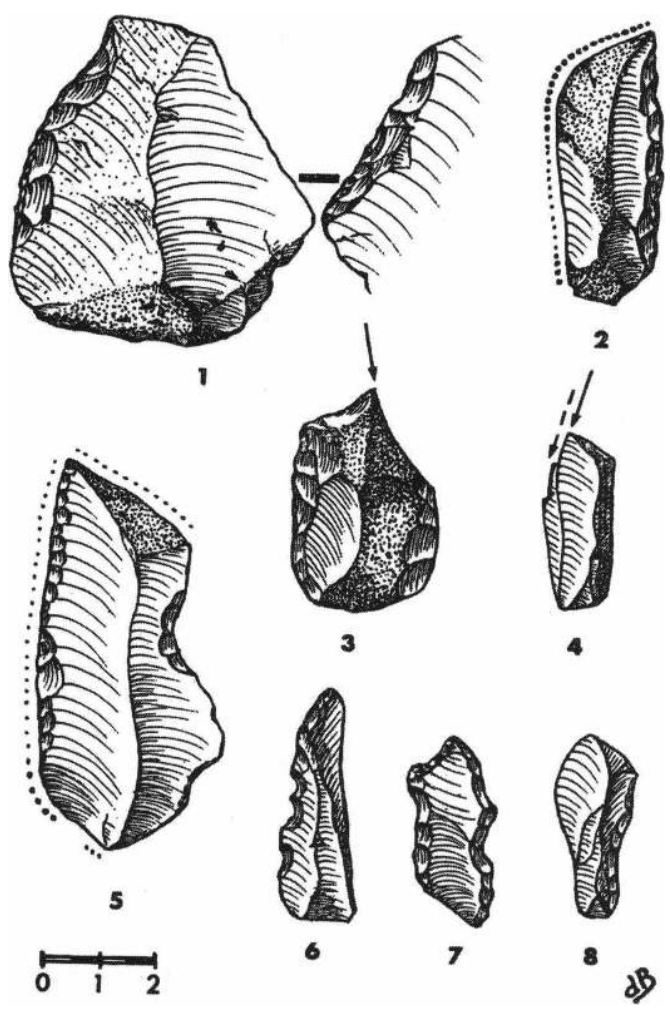

6 Contexte culturel sans référence anthropologique ni chronologique, n'étant en somme ni de tradition ibéromaurusienne, ni d'affiliation capsienne? sans rattachement possible, faute aussi de rayonnement géographique, dans l'état actuel de nos connaissances

Que sait-on des autres documents?

L'outillage osseux représenté par un tranchet à biseau oblique s'est mal conservé ; la parure attestée par une coquille marine percée de Turbo rugosus, comporte aussi des portions d'ocre rouge, il existe un fragment de galène, des graviers roulés et colorés, des tests d'œuf d'autruche non incisés.

9 Les restes d'une faune consommée ont été identifiés par F. Doumergue. Ce sont de fréquents ossements de taureaux sauvages (Bos primigenius), de l'antilope bubale (Alcelaphus boselaphus), d'antilope dorcas (Gazella dorcas), ou rares, d'Ovine (?), d'Equidé, d'Oiseaux, et de tortue terrestre (testudo). Quant aux gastéropodes terrestres (Helix) et aux bivalves d'eau douce (Unio), ils sont plutôt rares. Les ressources d'alors prélevées dans le paysage alentour restent à préciser, aucune documentation faunique et botanique, n'a été fournie par les sites voisins.

\section{Autonomie culturelle du Kérémien}

10 L'individualisation du Kérémien mise en lumière par J. Tixier résulte de la confrontation de trois ensembles lithiques de la région de Tiaret. Il s'agit encore du site de Kef el-Kérem, mais des récoltes de G. Vuillemot; du gisement de plein air du Bois de Pins et des fouilles et récoltes de R. de Bayle des Hermens (1959-1965) et du site de la Jumenterie, (fig. 1) avec les fouilles et récoltes de R. de Bayle des Hermens et J. Tixier 
(1967-1972). Cette abondante documentation confirme, avec quelques fluctuations, l'importance des grattoirs (entre 48,3 et 39,67\%), des lamelles à dos (entre 29,5 et $17,65 \%)$, et le maintien des racloirs (8,51\% au Kef el-Kérem). Elle souligne la variabilité des coches et denticulés (entre 2 et 17,24 \%). Dans le groupe des géométriques (3,53\%), les segments $(2,91 \%)$, souvent minuscules dominent à la Jumenterie. "Les courbes cumulatives dressées par J. Tixier pour les industries des trois gisements révèlent une identité d'autant plus remarquable que les procédés de récolte sont différents » (Camps, 1974 : 212).

\section{Conclusion}

11 En somme, même privé de repères stratigraphiques, chronologiques et environnementaux, le Kérémien lithique se singularise encore. L'aspect qualitatif de cet outillage est certes déroutant, en raison de ce que certains auteurs qualifient de «facture générale négligée », tenant à l'emploi de matières premières locales d'assez médiocre qualité de taille (calcédoine), en raison aussi d'un débitage "expédient " produisant beaucoup d'éclats. Mais on n'a pas encore compris pourquoi il en avait été ainsi. Quel était donc le projet d'activité de ces populations?

Sans se laisser indirectement influencer par des impressions esthétiques, ces traits contrastent beaucoup avec ceux qu'offrent les outillages ibéromaurusiens et capsiens.

Ce contraste dans le traitement de la matière lithique a contribué à écarter le Kérémien de filiations technologiques ibéromaurusienne ou capsienne, mais cet argument était-il vraiment discriminant?

Projet : il reste à comprendre et à valoriser en synergie des instruments et des activités. Les recherches futures s'appuieront sur une autre grille de lecture technique, distincte du tandem laminaire-lamellaire, ici inapproprié. Elles devront introduire dans leur démarche l'éclat et la grande lame comme supports recherchés. Ces recherches devront bénéficier d'un équipement d'investigation plus pénétrant. Il conviendra d'identifier quelques activités domestiques aux caractéristiques stables, en s'appuyant sur la mise en évidence de traces d'usure spécifiques, sur les bords et faces des grattoirs et des racloirs notamment, qui seront à reproduire expérimentalement. Ainsi parviendra-t-on à comprendre le rôle décisif du couple grattoirs-racloirs dans le Kérémien et celui de son cortège de macro-supports systématiquement transformés, répondant aux objectifs d'un artisanat singulier (en rapport avec le traitement des peaux, peut-être), annonçant vraisemblablement l'entrée dans le monde néolithique. Je pense qu'il y a dans ce couple documentaire une potentialité expressive qui n'a pas même été soupçonnée, et moins encore exploitée. Parce que l'optique des travaux d'alors a toujours conduit à rechercher des arguments en faveur d'une intégration du Kérémien dans l'ensemble culturel Epipaléolithique s.l., alors que le Kérémien, s'il s'avérait être un faciès autonome dans l'avenir, pourrait se déconnecter délibérément de l'Épipaléolithique et affirmer de nouveaux comportements annonciateurs d'une néolithisation. Voici une autre esquisse qui pourrait permettre de comprendre comment en région sub-atlasique occidentale se serait opéré le passage à la néolithisation.

\section{Remerciement}

15 Ce fut une très grande chance que de pouvoir rencontrer Monsieur Gustave vUILLEMOT à Paris, à l'Institut de Paléontologie Humaine. Il a pu m'exposer ce sujet Kérémien en 
ouvrant sa carte de la région. C'était un jour de novembre 2000, il a été un guide aux idées claires, cheminant en pensée dans les collines cartographiées. Je lui suis reconnaissante de m'avoir transmis quelque chose de chaleureux de son constant attachement pour cette région de l'Algérie et ses pionniers de la recherche préhistorique.

\section{BIBLIOGRAPHIE}

BALOUT L., 1955. « Préhistoire de l'Afrique du Nord. Essai de chronologie ». Paris, A.M.G, p. 345, 365-366. BAYLE DES HERMENS R. de, (1959), 1965. « La station préhistorique du Bois de Pins, route de TiaretTakdempt (Oranie) ». Actes du XVI Congrès Préhistorique de France, Monaco, p. 231-243.

BAYLE DES HERMENS R. de, et TIXIER J., (1967), 1972. « Le gisement Kérémien de la Jumenterie de Chaou Tiaret (Algérie) ». Actes du VIe Congrès Panafricain de Préhistoire de Dakar, p. 288-293.

BONNEAU G, et Vassot J., 1955. « Nouvelles stations préhistoriques de la région de Tiaret ». Libyca, t. III, p. 287-290.

CADENAT P., 1966. «Atlas préhistorique de l'Algérie. Feuille n 33 P. Tiaret ». Libyca, t. XIV, p. 21-213 (p. 94-96).

CADENAT P., (1969), 1972. « Le Kérémien. Quelques aspects particuliers de l'industrie ». Actes du XIX'Congrès Préhistorique de France, Auvergne, p. 128-133.

CADENAT P., et Vuillemot G, 1944. « La station préhistorique de Kef el-Kérem (Djebel Nador) ». Bull. de la Soc. de Géogr. et d'Archéol. d'Oran, t. LXV, p. 52-65.

CAMPS G., 1974. Les civilisations préhistoriques de l'Afrique du Nord et du Sahara. Paris, Doin, (p. 211-214).

DOUMERgue F.,1898. « Contribution au préhistorique de la Province d'Oran ». 27e Congrès de l'AFAS, Nantes, p. 574-583 (Djebel Mékaïdou, p. 577).

\section{INDEX}

Mots-clés : Epipaléolithique, Préhistoire 